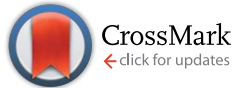

Cite this: RSC Adv., 2015, 5, 9722

Received 7th November 2014 Accepted 5th January 2015

DOI: 10.1039/c4ra14076a

www.rsc.org/advances

\section{Recovery rate and homogeneity of doping europium into luminescent metal hydrides by chemical analysis $\uparrow$}

\begin{abstract}
R. Hahn, ${ }^{a}$ N. Kunkel,,$^{\text {ab }}$ C. Hein, ${ }^{a}$ R. Kautenburger ${ }^{a}$ and H. Kohlmann ${ }^{\star b}$
During the investigation of concentration dependent properties of phosphors, such as emission intensities, the knowledge of the activator ion concentration is of great importance. Herein we present a study on recovery rate and homogeneity of the activator ion concentration by chemical analysis in luminescent europium doped metal hydrides. The analysis method was established on the model system $\mathrm{Eu}_{x} \mathrm{Sr}_{1-x} \mathrm{H}_{2}$ and applied to brightly emitting hydridic perovskites $\mathrm{LiMH}_{3}: \mathrm{Eu}^{2+}$ and $\mathrm{LiMD}_{3}: \mathrm{Eu}^{2+}(\mathrm{M}=\mathrm{Sr}, \mathrm{Ba})$. The nominal activator ion concentrations calculated from initial weights are in good agreement with those determined via ICP-MS for $10^{-3} \% \leq x$ both for the hydrides (deuterides) and the parent alloys. The synthesis protocol thus allows a reliable method for reproducibly doping europium into metal hydrides. Luminescence spectra of $\mathrm{LiEu}_{x} \mathrm{Sr}_{1-x} \mathrm{H}_{3}$ with $x=0.0037 \%$ show a broad band emission at room temperature typical for $\mathrm{Eu}^{2+} 4 \mathrm{f}^{6} 5 \mathrm{~d}^{1}-4 \mathrm{f}^{7}$ transitions. Below $80 \mathrm{~K}$, a vibronic fine structure is observed with vibrational coupling frequencies of approximately 100,370 and $970 \mathrm{~cm}^{-1}$.
\end{abstract}

\section{Introduction}

Inorganic luminescent materials play an important role in efficient energy conversion and contribute to saving energy for lighting purposes. Europium is being used for instance in efficient blue-emitting $\mathrm{BaMgAl}_{10} \mathrm{O}_{17}$ : $\mathrm{Eu}(\mathrm{II})$ and red-emitting $\mathrm{Y}_{2} \mathrm{O}_{3}$ : $\mathrm{Eu}(\mathrm{III})$ in LEDs and plasma screens. ${ }^{\mathbf{1 - 3}}$ The electric-dipole and parity-allowed $4 \mathrm{f}^{7}-4 \mathrm{f}^{6} 5 \mathrm{~d}$ transition in Eu(II) produces high quantum yields and thus high emission intensities. In contrast to trivalent europium with an $\mathrm{f}-\mathrm{f}$ transition $\mathrm{Eu}(\mathrm{II})$ further exhibits a strong dependence of the emission wavelength on the coordination of the activator ion. Eu(II) in oxide and halide host lattices often emits blue or green light, ${ }^{2}$ while more polarisable ions like sulfides and nitrides induce emission in the green to red colour region. ${ }^{2,4}$ Luminescent materials usually contain only small amounts of the activator ion and for efficient host lattices luminescence can often be observed even for ppm amounts of the activator ion. Many investigations have been carried out to study concentration dependencies of the luminescence intensity. Exact control of the activator ion concentration is of great importance, since luminescence properties may be strongly

anorganic Solid State Chemistry, Saarland University, P. O. Box 151150, 66041 Saarbrücken, Germany

${ }^{b}$ Inorganic Chemistry, University Leipzig, Johannisallee 29, 04103 Leipzig, Germany. E-mail: holger.kohlmann@uni-leipzig.de

$\dagger$ Electronic supplementary information (ESI) available: High resolution spectrum of $\mathrm{LiSrH}_{3}: \mathrm{Eu}^{2+} 0.0037 \mathrm{~mol} \%$ at $4 \mathrm{~K}$ and assignment of the vibronics. See DOI: $10.1039 / \mathrm{c} 4 \mathrm{ra} 14076 \mathrm{a}$

\$ Current institution: Chimie Paristech-CNRS, Institut de Recherche de Chimie Paris, 11 rue Pierre et Marie Curie, 75005 Paris, France. influenced. For instance, too high concentrations often lead to energy transfer and consequently to luminescence quenching. ${ }^{5}$ However, analyses of the exact content of the activator ion or the local coordination environment via a method different from luminescence spectroscopy are rather scarce and concentrations are usually calculated according to initial weights. Lately, an NMR and ESR study regarding the $\mathrm{Nd}^{3+}$ and $\mathrm{Gd}^{3+}$ content in $\mathrm{LaPO}_{4}$ was carried out, studying doping rates down to approximately 0.1 at\%. ${ }^{6}$

Chemical analyses of europium species in solution via inductively-coupled plasma-mass spectrometry (ICP-MS) are commonly used, e. g. in rock samples, ${ }^{7}$ in water, ${ }^{\mathbf{8 9}}$ in fine chemicals, ${ }^{\mathbf{1 0}}$ in biological samples ${ }^{\mathbf{1 1 , 1 2}}$ or in non-radioactive model systems using europium as chemical homologue for the highly toxic americium (as one of the main components in radioactive waste) to analyse the complexation behavior of europium with natural organic matter ${ }^{\mathbf{1 3}, \mathbf{1 4}}$ as well as the sorption/desorption properties in pore water/clay systems. ${ }^{15}$

Another feature often neglected concerns the homogeneity of the activator ions within the host materials. In a recent study of $\mathrm{Eu}(\mathrm{II})$ in a metal-organic framework it was shown that at least for higher doping rates $(20 \%)$ on a nanometer to low micrometer scale the europium content varied between $12-28 \%$, proving a considerable heterogeneity. ${ }^{16}$

Recently, we have studied $\mathrm{Eu}^{2+}$ luminescence in alkaline earth metal hydrides ${ }^{17}$ and perovskite type hydrides. ${ }^{18,19}$ In order to check whether the europium concentrations of our luminescent materials calculated based on the initial weights are in accordance with the real europium content and whether europium is distributed homogeneously within the host lattice, we 
present a method of analysis of the phosphors using the model system $\mathrm{Eu}_{x} \mathrm{Sr}_{1-x} \mathrm{H}_{2}$ within this work. $\mathrm{EuH}_{2}$ and $\mathrm{SrH}_{2}$ are isotypic and the existence of a solid solution series was shown before. ${ }^{17}$ In strontium-rich samples a broad band emission at $728 \mathrm{~nm}$ was observed and assigned to the $\mathrm{Eu}^{2+} 4 \mathrm{f}^{7}-4 \mathrm{f}^{6} 5 \mathrm{~d}$ transition. The system was chosen due to its relative simplicity and we will also show that the methods can be applied for ternary systems such as the yellow emitting $\mathrm{LiSrH}_{3}: \mathrm{Eu}^{2+} .^{18}$

Spectra of $\mathrm{SrH}_{2}, \mathrm{LiSrH}_{3}$ and $\mathrm{LiBaH}_{3}$ with relatively high doping rates can be found in. ${ }^{17,18} \mathrm{SrH}_{2}$ samples with relatively low doping rates do not show emission, because emission intensities of alkaline earth metal hydrides are rather low. However, $\mathrm{LiSrH}_{3}: \mathrm{Eu}^{2+}$ shows a bright emission even at very low doping rates and herein we also present emission spectra of a low doped sample whose europium concentration was determined during our study.

\section{Experimental details}

\section{Sample preparation}

Due to their air sensitivity, all solids were handled in an argon filled glove box. The alloys $\mathrm{Eu}_{x} \mathrm{Sr}_{1-x}$ were synthesised by melting reactions of the elements (Eu ingot Alfa Aesar 99.9\%, Sr pieces, distilled, Alfa Aesar 99.9\% and Kristallhandel Kelpin, all mechanically surface cleaned before use) in niobium ampoules in a high-frequency furnace and hydrogenated in an autoclave at $650 \mathrm{~K}$ and approx. 100 bar $\mathrm{H}_{2}$ pressure $\left(\mathrm{H}_{2}\right.$ Praxair 99.9\%) for about 2-3 days. The autoclave and crucibles consist of the hydrogen resistant Nicrofer® 5219 alloy (Inconel 718). Preparation of samples $\mathrm{Eu}_{x} \mathrm{Sr}_{1-x}$ with $x<0.05$ was performed by melting alloys with higher europium concentration with corresponding amounts of strontium metal. A list of the initial weights is given in the ESI. $\uparrow$ The ternary hydrides were prepared as described in. ${ }^{18,19}$ Note that therein the europium concentrations are given with respect to the substitutional site $\mathbf{M}$ in $\mathrm{AMH}_{3}$, therefore $0.5 \%$ corresponds to an overall value of $0.25 \%$ in our present study. For ICP-MS measurements, three to four samples were taken from each intermetallic phase in order to assure representative sampling and to probe homogeneity, but only one sample from each hydride.

\section{X-ray powder diffraction}

X-ray powder diffraction data were collected on a PANalytical Philips X'Pert Pro with focusing Bragg-Brentano geometry and a fine focus $\mathrm{X}$-ray tube $\left(\mathrm{CuK} \alpha_{1,2}\right.$ radiation). The ratio of $\mathrm{CuK} \alpha_{1}$ and $\mathrm{K} \alpha_{2}$ was determined using a silicon standard (NIST640d). Finely ground samples were enclosed between kapton foils with apiezon grease. The data collection time was $60 \mathrm{~min}$ and the diffraction range $10^{\circ} \leq 2 \theta \leq 110^{\circ}$. Crystal structures were refined using the program TOPAS 4.2 (Bruker AXS, Karlsruhe, Germany) ${ }^{\mathbf{2 0}}$ together with the fundamental parameter approach. ${ }^{21}$ The instrumental function was determined empirically by means of a reference scan of $\mathrm{LaB}_{6}$. Typically, complete crystal structures including hydrogen positions were taking as starting structures from literature and scale factors, lattice parameters, zero point shift,

thermal displacement and microstructural parameters were refined.

\section{Chemicals and standards for ICP-MS}

The quality of all chemicals used was analytical reagent grade or better (e.g. suprapure) from Merck. All solutions were prepared with MilliQ deionised water (18.2 M 2 , MilliQ-System, Millipore, Schwalbach, Germany). Single element standards of europium and holmium Certipur® from Merck were used and Argon 5.0 (99.999\%, Praxair) as plasma gas.

\section{ICP-MS measurements}

For calibration a 1000 ppm europium stock solution was prepared by dissolving europium metal in $\mathrm{HNO}_{3}(3.25 \%)$ and diluting to a concentration of 1 ppm europium. $10 \mu \mathrm{L}$ of a 10 ppm holmium solution as internal standard was added to correct time dependent sensitivity deviation of the ICP-MS system to the calibration samples as well as all other samples. Additionally, $300 \mu \mathrm{L}$ of $\mathrm{HNO}_{3}$ (65\%, suprapure) were added to prohibit metal sorption onto the sample container surface. MilliQ water was then added to obtain the different europium concentrations (5-500 ppb).

All samples were first dissolved in $1 \mathrm{~mL} \mathrm{HNO}_{3}$ (3.25\%) and stocked up to a concentration of $1000 \mathrm{ppm}$ (according to initial weights of the samples). Samples with europium concentration of $7 \times 10^{-5}-7 \times 10^{-7} \%$ were diluted with a total sample dilution of 1 : 3 using an ICP-MS solution $\left(300 \mathrm{~mL} \mathrm{HNO}_{3} 65 \%, 1 \mathrm{~mL}\right.$ internal standard, $636 \mathrm{~mL}$ MilliQ water). To all other samples 10 $\mu \mathrm{L}$ of internal standard and $300 \mu \mathrm{L}$ of $\mathrm{HNO}_{3}(65 \%)$ were added and stocked up with MilliQ water to reach a volume of $10 \mathrm{~mL}$. $9.5 \mathrm{mg}$ of the pure strontium samples were dissolved in $5 \mathrm{~mL}$ $\mathrm{HNO}_{3}(3.25 \%) .10 \mu \mathrm{L}$ of internal standard, $300 \mu \mathrm{L}$ of $\mathrm{HNO}_{3}$ (65\%) and MilliQ water were added to reach a volume of $10 \mathrm{~mL}$.

An Agilent 7500cx ICP-MS (Agilent Technologies, Santa Clara, USA) was used for the isotopic measurements; detailed analytical conditions are given in Table 1 . A correction of the isotope intensities via normalisation by the intensity of ${ }^{165} \mathrm{Ho}$ as internal standard was carried out and samples were measured in triplicate. ${ }^{15} \mathrm{RSD}$ values are in the range of 0.1 and $2.2 \%$.

Table 1 Operating parameters and analytical conditions of the ICPMS

\begin{tabular}{ll}
\hline ICP-MS & Agilent $7500 \mathrm{cx}$ \\
\hline RF-power & $1550 \mathrm{~W}$ \\
Cooling/auxiliary gas & $15.0 / 1.05 \mathrm{~L} \mathrm{~min}^{-1}$ \\
Dwell times & $300 \mathrm{~ms}(3 \times 100 \mathrm{~ms})$ per mass \\
Repetition & 3 times \\
\hline
\end{tabular}

Samples

Ho-ICP standard Analysed isotope Internal standard Detection limit

\author{
Certipur® (Merck) \\ ${ }^{153} \mathrm{Eu}$ \\ ${ }^{165} \mathrm{Ho}$ \\ 10 ppt (ng kg $\left.{ }^{-1}\right)$
}




\section{Luminescence measurements}

Temperature dependent photoluminescence emission spectra and lifetime measurements were carried out on an Edingburgh FLS-920 spectrofluorometer with a pulsed $376.8 \mathrm{~nm}$ Edingburgh laser diode for sample excitation, a monochromator according to Czerny-Turner (500 nm focal length) for emission, a R928P (Hamamatsu) photomultiplier tube for detection of the emission spectra and a H7422-02 photomultiplier tube (Hamamatsu) for detection of the decay curves. Low temperature measurements were carried out using an Oxford liquid helium cold finger. Due to moisture and air sensitivity, samples were enclosed in sealed silica tubes of $0.5-1 \mathrm{~cm}$ diameter and they were attached to the cold finger using high purity silver paint in order to facilitate heat transfer and minimise the lagging of real temperatures behind the nominal ones assigned to the spectra. Spectra were corrected for lamp intensity and photomultiplier sensitivity. Luminescence decay curves were recorded under pulsed excitation with the Edinburgh $376.8 \mathrm{~nm}$ pulsed diode laser. Neglecting a slight deviation from single exponential behavior in the higher temperature range (faster initial decay), a single exponential function was used for the analysis of the luminescence decay curves.

\section{Results and discussion}

Melting of the elements produced alloys $\mathrm{Eu}_{x} \mathrm{Sr}_{1-x}$ with a silver luster, which were hydrogenated to yield metal hydrides $\mathrm{Eu}_{x} \mathrm{Sr}_{1-x} \mathrm{H}_{2}$ as fine powders. For samples with $0.5 \% \leq x \leq 2 \%$ yellow to orange colors were observed, whereas samples with lower europium concentrations were colorless. Samples were single-phase according to X-ray powder diffraction and Rietveld refinement (Fig. 1). Due to the low doping concentrations and like ionic radii of $\mathrm{Sr}^{2+}$ and $\mathrm{Eu}^{2+}$ refined lattice parameters vary very little and cannot reflect the concentration of $\mathrm{Eu}^{2+}$ properly given the accuracy of a routine X-ray powder diffraction measurement (Table 2).

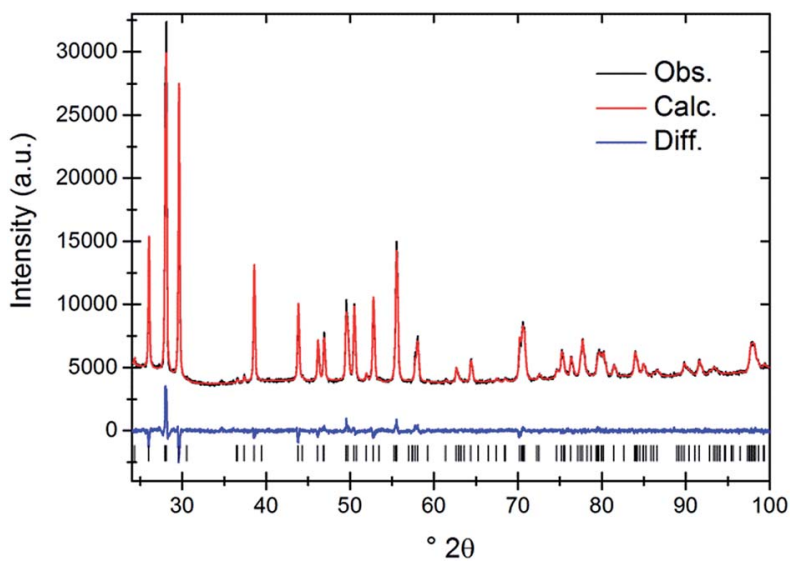

Fig. 1 Rietveld refinement of the crystal structure of $\mathrm{SrH}_{2}$ : $\mathrm{Eu}^{2+}(2 \%)$. Observed (red line), calculated (black line), and difference (observedcalculated; bottom, blue line) $\mathrm{X}$-ray powder diffraction patterns $(\lambda=1.54056 \AA)$. Markers indicate Bragg peak positions of $\mathrm{SrH}_{2}: \mathrm{Eu}^{2+}$ $(2 \%)$.
Table 2 Lattice parameters in $\mathrm{Eu}_{x} \mathrm{Sr}_{1-x} \mathrm{H}_{2}$ as determined by Rietveld refinement on $\mathrm{X}$-ray powder diffraction data

\begin{tabular}{llll}
$\begin{array}{l}\text { Nominal } x \text { in } \\
\mathrm{Eu}_{x} \mathrm{Sr}_{1-x} \mathrm{H}_{2} \text { in } \%\end{array}$ & $a / \mathrm{pm}$ & $b / \mathrm{pm}$ & $c / \mathrm{pm}$ \\
\hline 2 & $638.164(9)$ & $387.344(5)$ & $732.71(1)$ \\
1 & $638.357(7)$ & $387.518(4)$ & $732.632(8)$ \\
0.5 & $638.456(8)$ & $387.505(5)$ & $732.650(9)$ \\
0.05 & $638.456(1)$ & $387.517(7)$ & $732.81(1)$ \\
0.005 & $638.36(1)$ & $387.537(6)$ & $732.57(1)$ \\
0.0005 & $638.38(1)$ & $387.582(9)$ & $732.45(2)$ \\
$10^{-5}$ & $638.38(1)$ & $387.61(1)$ & $732.68(2)$ \\
$10^{-6}$ & $638.51(2)$ & $387.45(1)$ & $733.19(2)$ \\
$10^{-7}$ & $638.31(1)$ & $387.618(8)$ & $732.29(2)$
\end{tabular}

Chemical analysis of the dissolved starting materials by ICPMS gave 0.225 ppm europium in strontium from Alfa Aesar (99.9\%) and $0.01 \mathrm{ppm}$ europium in the strontium rod from Kristallhandel Kelpin. Thus samples $\mathrm{Eu}_{x} \mathrm{Sr}_{1-x}$ and $\mathrm{Eu}_{x} \mathrm{Sr}_{1-x} \mathrm{H}_{2}$ with europium contents $x \leq 5 \times 10^{-2} \%$ were prepared from the latter material. As a consequence of the $0.01 \mathrm{ppm}$ europium in the strontium metal europium in $\mathrm{Eu}_{x} \mathrm{Sr}_{1-x}$ and $\mathrm{Eu}_{x} \mathrm{Sr}_{1-x} \mathrm{H}_{2}$ samples the lowest reasonably achievable doping content was $10^{-6} \%$.

Analyses of alloys $\mathrm{Eu}_{x} \mathrm{Sr}_{1-x}$ show differences in europium content up to a factor of 2.8 between samples taken from different spots on the same alloy (Table 3). This hints at some inhomogeneity in the distribution of europium in strontium, which is not unexpected. Such inhomogeneity in the alloys most probably will cause inhomogeneity in the hydrides as well, which could not be measured, however, due to decrepitation of the alloys to a fine powder upon hydrogenation.

The relative deviation of europium content as determined by ICP-MS from the nominal contents in the metal hydrides $\mathrm{Eu}_{x} \mathrm{Sr}_{1-x} \mathrm{H}_{2}$ is only $6 \%$ for $x=0.5 \%$ and increases with decreasing europium content up to $70 \%$ for $x=5 \times 10^{-4} \%$ (Table 3). Samples with lower europium content were analysed as well; however, the concentrations found for those samples lie within the same range as the blank samples and are therefore not reliable. At least for the higher contents shown in Tables 2 and 3, which are in the range of conventional activator contents in phosphors, the correspondence between nominal and experimentally determined europium contents is satisfactory (Fig. 2 and 3). Nominal europium contents are thus a reasonable measure of the actual content at least for $10^{-3} \% \leq x$, where the deviations were found to be between $6 \%$ and $32 \%$.

The analysis of the ternary luminescent materials yielded the following europium concentrations (see Table 4).

Again, the agreement between nominal and experimentally observed values is satisfactory. Nominal values based on the initial weights may therefore be regarded as a reasonable measure for the true activator content in europium doped metal hydrides prepared by melting of the metals and subsequent hydrogenation, even for very low dopant concentrations; however, europium distributions in powder samples may be inhomogeneous. This might lead to varying local environments and thus broadening of absorption and emission bands. 
Table 3 Nominal and experimentally determined europium contents $x$ of alloys $\mathrm{Eu}_{x} \mathrm{Sr}_{1-x}$ and their hydrides Eu $\mathrm{Sr}_{1-x} \mathrm{H}_{2}$ in \%

Europium content $x / \%$

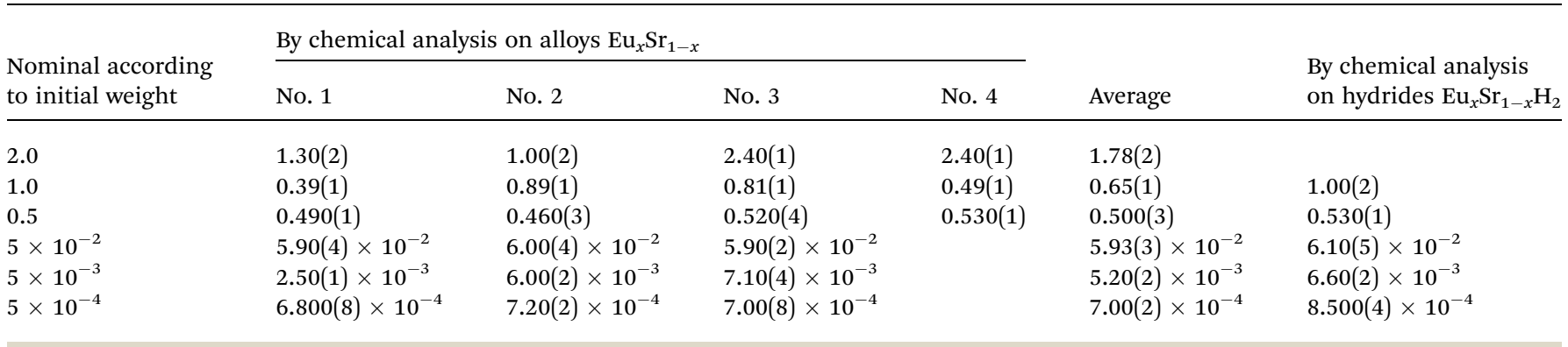

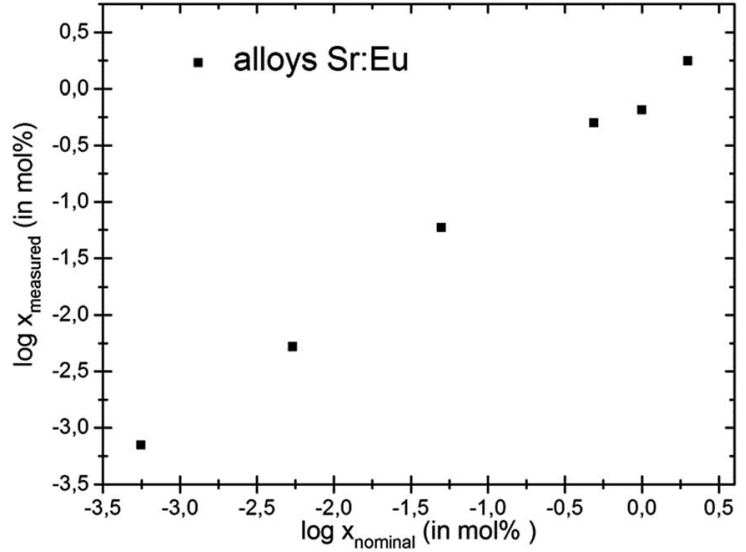

Fig. 2 Log-log plot showing the relation between the nominal and real europium contents in the intermetallic phases $\mathrm{Eu}_{x} \mathrm{Sr}_{1-x}$.

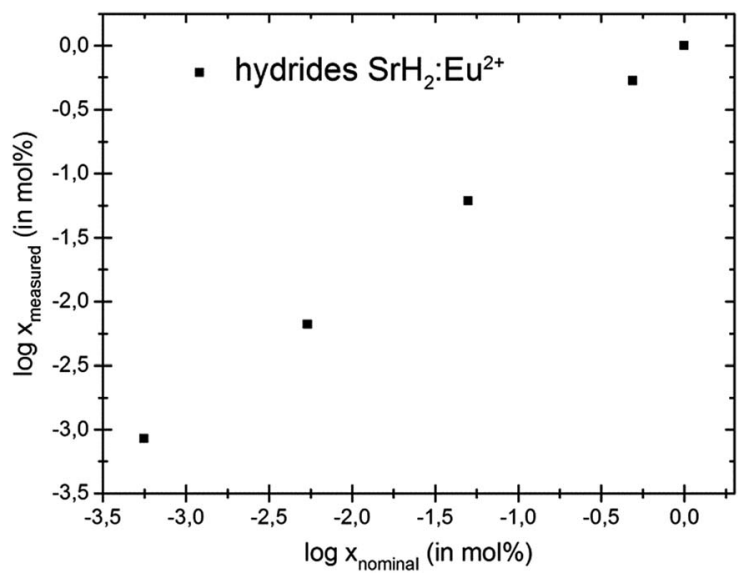

Fig. 3 Log-log plot showing the relation between nominal and real europium contents in the hydrides $\mathrm{Eu}_{x} \mathrm{Sr}_{1-x} \mathrm{H}_{2}$.

In contrast to higher doped $\mathrm{LiSrH}_{3}$ samples, which are of a light yellow colour, $\mathrm{LiSrH}_{3}: \mathrm{Eu}^{2+} 0.0037 \%$ is colourless. However, in spite of the low europium content it still shows a bright yellow emission as described for samples with higher doping rates. ${ }^{18}$ In Fig. 4 temperature dependent spectra of $\mathrm{LiSrH}_{3}: \mathrm{Eu}^{2+} 0.0037 \%$ are given. In good agreement with, ${ }^{18}$ the spectra show a broad band emission at room temperature which is typically observed for $\mathrm{Eu}^{2+} 4 \mathrm{f}^{6} 5 \mathrm{~d}^{1}-4 \mathrm{f}^{7}$
Table 4 Europium content in ternary europium doped metal hydrides $\mathrm{LiMH}_{3}$ and $\mathrm{LiMD}_{3}(\mathrm{M}=\mathrm{Sr}, \mathrm{Ba})$

\begin{tabular}{lll}
\hline Compound & $\begin{array}{l}\text { Nominal europium } \\
\text { content } x / \%\end{array}$ & $\begin{array}{l}\text { Europium content } x \\
\text { determined by ICP-MS/\% }\end{array}$ \\
\hline $\mathrm{LiSrH}_{3}: \mathrm{Eu}^{2+}$ & 0.247 & $0.268(3)$ \\
$\mathrm{LiSrD}_{3}: \mathrm{Eu}^{2+}$ & 0.240 & $0.26(3)$ \\
$\mathrm{LiBaH}_{3}: \mathrm{Eu}^{2+}$ & 0.248 & $0.27(2)$ \\
$\mathrm{LiBaD}_{3}: \mathrm{Eu}^{2+}$ & 0.217 & $0.23(4)$ \\
$\mathrm{LiSr}_{0.8} \mathrm{Ba}_{0.2} \mathrm{H}_{3}: \mathrm{Eu}^{2+}$ & 0.239 & $0.25(2)$ \\
$\mathrm{LiSrH}_{3}: \mathrm{Eu}^{2+}$ & 0.005 & $0.0037(0)$
\end{tabular}

emission and below approximately $80 \mathrm{~K}$, a vibronic fine structure is observed. Due to the low europium content, this fine structure shows a slightly better resolution than that of the higher concentrated samples and higher modes are still clearly visible and can be assigned. Thus it may be concluded that the spectral resolution may be improved by the use of lower doping rates; if the emission intensities allow for it. The vibrational energies with respect to the zero phonon line $0-0-0$ are listed in the ESI, $\uparrow$ Table 1 and the assignment of the bands was done according to. ${ }^{18} \mathrm{~A}$ vibrational coupling with three different modes is observed (approx. 100, 370 and $970 \mathrm{~cm}^{-1}$ ).

The temperature dependence of the lifetimes is shown in Fig. 5. In good agreement with earlier results,${ }^{18}$ the quenching temperature is approximately $150 \mathrm{~K}$.

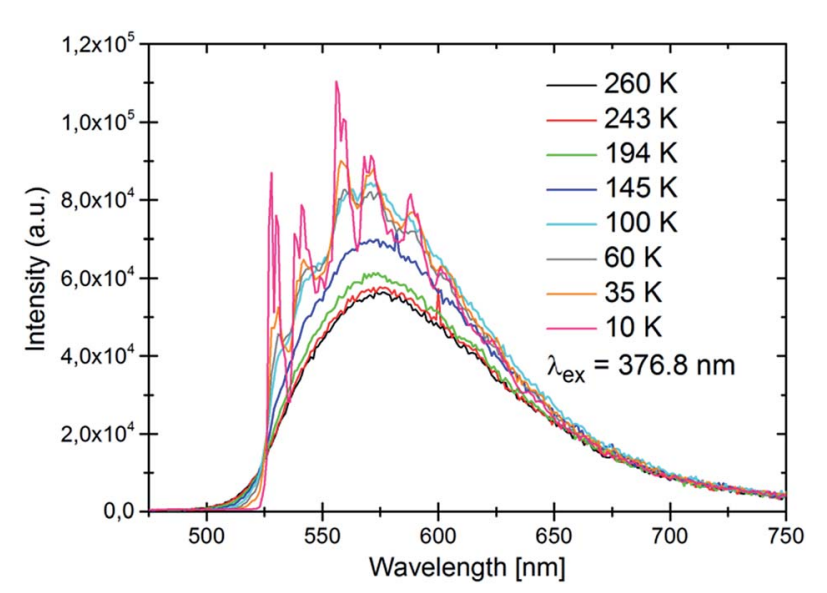

Fig. 4 Temperature dependent emission spectra of $\mathrm{LiSrH}_{3}: \mathrm{Eu}^{2+}$ $0.0037 \mathrm{~mol} \%$ under UV excitation (376.8 nm). 


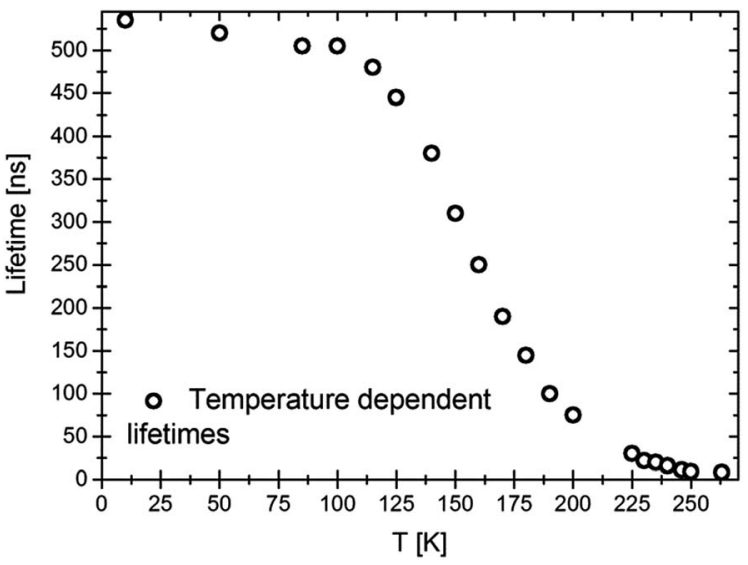

Fig. 5 Temperature dependence of the lifetimes of $\mathrm{LiSrH}_{3}: \mathrm{Eu}^{2+}$ $0.0037 \mathrm{~mol} \%$. Excitation at $376.8 \mathrm{~nm}$, emission at $580 \mathrm{~nm}$.

With about $530 \mathrm{~ns}$, the lifetime at $10 \mathrm{~K}$ is slightly larger than for a higher europium concentration (0.25 mol\%, approximately $420 \mathrm{~ns}$ ). This indicates, together with the slightly better resolved vibronics, that in higher doped samples concentration effects might already play a role. It is well known that higher doping rates may lead to concentration quenching and a shortening of the observed lifetimes. ${ }^{5}$

\section{Conclusion}

The exact knowledge of the doping concentration and homogeneity of the activator ion in luminescent samples is of great importance for concentration dependent effects such as emission intensities. Chemical analysis on europium doped alloys and their metal hydrides was performed by ICP-MS. Nominal activator ion concentrations calculated from initial weights in $\mathrm{Eu}_{x} \mathrm{Sr}_{1-x}$ and $\mathrm{Eu}_{x} \mathrm{Sr}_{1-x} \mathrm{H}_{2}$ are in good agreement with experimentally determined concentrations for $10^{-3} \% \leq x$. The synthesis protocol thus allows a reliable method for reproducibly doping europium into metal hydrides. A good agreement between nominal and experimental values was also achieved for compounds $\mathrm{LiMH}_{3}: \mathrm{Eu}^{2+}$ and $\mathrm{LiMD}_{3}: \mathrm{Eu}^{2+}(\mathrm{M}=\mathrm{Sr}, \mathrm{Ba})$ at dopant levels around $0.25 \%$ and at $0.0037 \%$ for $\mathrm{LiSrH}_{3}: \mathrm{Eu}^{2+}$. For the latter luminescence spectra show a broad band emission at room temperature which is typically observed for $\mathrm{Eu}^{2+} 4 \mathrm{f}^{6} 5 \mathrm{~d}^{1}-4 \mathrm{f}^{7}$ transitions. Below $80 \mathrm{~K}$, a vibronic fine structure is observed with vibrational coupling frequencies of approximately 100, 370 and $970 \mathrm{~cm}^{-1}$.

\section{Acknowledgements}

We are grateful to the Deutsche Forschungsgemeinschaft (DFG grant KO1803/7-1) for financial support. N.K. and H.K. would like to thank Prof. Andries Meijerink and M. Sc. Tim Senden for technical support and the Landesgraduiertenförderung Saar for a doctoral scholarship.

\section{Notes and references}

1 H. Höppe, Angew. Chem., Int. Ed., 2009, 48, 3572.

2 P. Dorenbos, J. Lumin., 2003, 104, 239.

3 T. Jüstel, H. Nikol and C. Ronda, Angew. Chem., Int. Ed., 1998, 37, 3084.

4 P. Pust, A. S. Wochnik, E. Baumann, P. J. Schmidt, D. Wiechert, C. Scheu and W. Schnick, Chem. Mater., 2014, 26, 3544.

5 A. Huignard, T. Gacoin and J.-P. Boilot, Chem. Mater., 2000, 12, 1090.

6 S. Maron, G. Dantelle, T. Gacoin and F. Devreux, Phys. Chem. Chem. Phys., 2014, 16, 18788.

7 N. N. Fedyunina, I. F. Seregina, M. A. Bolshov, O. I. Okina and S. M. Lyapunov, Anal. Chim. Acta, 2012, 713, 97.

8 K. J. Stetzenbach, M. Amano, D. K. Kreamer and V. F. Hodge, Ground Water, 1994, 32, 976.

9 W. Zhu, E. W. B. de Leer, M. Kennedy and G. J. F. R. Alaerts, Fresenius. J. Anal. Chem., 1998, 360, 74.

10 W. R. Pedreira, J. E. S. Sarkis, C. A. da Silva Queiroz, C. Rodrigues, I. A. Tomiyoshi and A. Abrao, J. Solid State Chem., 2003, 171, 3.

11 Y. Ming and L. Bing, Spectrochim. Acta, Part B, 1998, 53, 1447. 12 B. Li, Y. Sun and M. Yin, J. Anal. At. Spectrom., 1999, 14, 1843.

13 C. Möser, H. P. Beck and R. Kautenburger, Electrophoresis, 2012, 33, 1482.

14 R. Kautenburger, C. Hein, J. M. Sander and H. P. Beck, Anal. Chim. Acta, 2014, 816, 50.

15 R. Kautenburger and H. P. Beck, J. Environ. Monit., 2010, 12, 1295-1301.

16 J.-C. Rybak, M. Hailmann, P. R. Matthes, A. Zurawski, J. Nitsch, A. Steffen, J. G. Heck, C. Feldmann, S. Gotzendorfer, J. Meinhardt, G. Sextl, H. Kohlmann, S. J. Sedlmaier, W. Schnick and K. Müller-Buschbaum, J. Am. Chem. Soc., 2013, 135, 6896.

17 N. Kunkel, H. Kohlmann, A. D. Sayede and M. Springborg, Inorg. Chem., 2011, 50, 5873.

18 N. Kunkel, A. Meijerink and H. Kohlmann, Phys. Chem. Chem. Phys., 2014, 16, 4807.

19 N. Kunkel, A. Meijerink, M. Springborg and H. Kohlmann, J. Mater. Chem. C, 2014, 2, 4799.

20 Bruker, TOPAS V4.2, General profile and structure analysis software for powder diffraction data, User's Manual, AXS, Karslruhe.

21 A. A. Coelho, J. Appl. Crystallogr., 2003, 36, 86. 